\title{
Influence of Sternotomy on Delta Pulse Pressure and Delta Down During Open Chest Cardiac Surgery: A Preliminary Study
}

\author{
Eric Deflandre, MD, PhD, FCCP, FAHA ${ }^{*, \dagger, \ddagger, \S 1}$, \\ Simon Delande, MD ${ }^{\Uparrow}$, Jerome Cauvain, MD ${ }^{\Uparrow}$, \\ Pierre Geradon, $\mathrm{MD}^{\Uparrow}$, Anne-Francoise Donneau, $\mathrm{PhD}^{\|}$, \\ Simon Lacroix, MD*, Michel Van Dyck, MD ${ }^{\top}$ \\ *Department of Anesthesia, Clinique Saint-Luc de Bouge, Namur, Belgium \\ ${ }^{\dagger}$ Department of Ambulatory Surgery, Clinique Saint-Luc de Bouge, Namur, Belgium \\ ${ }^{\ddagger}$ University of Liege, Liege, Belgium \\ ${ }^{\S}$ Medical ASTES, Jambes, Belgium \\ "Department of Anesthesia, Catholic University of Louvain, Brussels, Belgium \\ "Biostatistics Unit, Department of Public Health, University of Liege, Liege, Belgium
}

Objective: Delta pulse pressure and delta down are used as dynamic preload indicators of fluid responsiveness during closed chest surgery. There are few data regarding their accuracy in open chest surgery. The present study aimed to evaluate the influence of sternotomy on the accuracy of both delta pulse pressure and delta down.

Design: Prospective study.

Setting: Single institution, nonacademic hospital.

Participants: The study comprised 127 adult patients scheduled for elective open chest cardiac surgery.

Interventions: Delta pulse pressure and delta down were calculated for all patients before and 10 minutes after sternotomy.

Measurements and Main Results: Statistical analyses were performed to assess the influence of sternotomy on the accuracy of delta down and delta pulse pressure. Mann-Whitney and Bland-Altman analyses demonstrated a significant influence of sternotomy on delta pulse pressure values but not on delta down values. Among patients who had a positive delta down and/or delta pulse pressure before sternotomy, sternotomy significantly modified the delta pulse pressure value $(\mathrm{p}=0.02)$, but not the delta down value $(\mathrm{p}=0.22)$. The kappa coefficient indicated a very good agreement between delta down before and after sternotomy (0.83) and a fair agreement between delta pulse pressure before and after sternotomy (0.4). The difference between kappa coefficients was highly significant $(\mathrm{p}<0.001)$.

Conclusions: Within the study population, sternotomy significantly influenced delta pulse pressure but not delta down. In this preliminary study, delta down appeared to be more accurate to evaluate fluid responsiveness during open chest surgery than did delta pulse pressure. Before promoting delta down in current practice, confirmation is needed on a larger scale.

(C) 2018 Elsevier Inc. All rights reserved.

Key Words: delta down; delta pulse pressure; monitoring; sternotomy; open chest surgery

This study is registered at ClinicalTrial.gov (NCT03047850), and preliminary results were presented at the American Society of Anesthesiologists annual meeting in October 2017 in Boston, MA.

${ }^{1}$ Address reprint requests to Eric Deflandre, MD, PhD, FCCP, FAHA, Chaussee de Tongres, 29, B-4000 Liege, Belgium.

E-mail address: eric.deflandre@gmail.com (E. Deflandre).
Optimization of intraoperative fluid administration improves outcome after surgery. ${ }^{1-4}$ To achieve this optimization, dynamic preload indicators offer better accuracy than do static preload indicators, such as central venous pressure or pulmonary capillary wedge pressure. ${ }^{5,6}$ Delta down (DD), delta pulse pressure (DPP), and stroke volume variation have been 
proposed as dynamic indicators of fluid responsiveness. ${ }^{5}$ They are validated in intensive care and in the operating room for closed chest procedures. $^{7-9}$ At a threshold value of $\geq 13 \%$, DPP has a sensitivity of $94 \%$ and a specificity of $96 \%$ in assessing hypovolemia. ${ }^{7}$ The authors have demonstrated that DD, with a threshold value of $5 \mathrm{mmHg}$, is as efficient as DPP for assessing hypovolemia in patients undergoing intracranial surgery. ${ }^{9}$

Several criteria have been described for the application of DD and DPP —adult patients ( $\geq 18$ y), patients must be mechanically ventilated (volume controlled mode and with a minimum of $8 \mathrm{~mL} / \mathrm{kg}$ tidal volume), patients must be in regular sinus rhythm, and the patient's thorax must be closed. ${ }^{5}$

DD and DPP now are widely used for perioperative fluid optimization in closed chest surgery. Nevertheless, the utility of these dynamic measures of fluid responsiveness remains unclear in patients undergoing sternotomy. This point needs to be clarified because open chest surgeries could be a challenge for anesthesiologists concerning fluid optimization, especially because numerous open chest procedures still are performed without transesophageal echography.

Some authors have investigated DPP under open thoracic conditions. ${ }^{10-13}$ Conversely, none of these studies evaluated the agreement between DPP values before and after sternotomy without any change in volemia. DD has never been assessed in open chest surgery.

The present study aimed to analyze the effect of sternotomy on the accuracy of DPP and DD values. This study was not designed to validate the use of DPP or DD after a fluid challenge in hypovolemic patients.

\section{Methods}

The study was approved by the Institutional Ethics Committee (Clinique Saint-Luc of Bouge, Namur, Belgium; reference number CE SLBO 2017/02). Because this study was limited to a data analysis, the Institutional Review Board waived the need for written informed consent. The trial was registered before patient enrollment at ClinicalTrial. gov (NCT03047850). The article adheres to the applicable Transparent Reporting of Evaluations with Nonrandomized Designs (TREND) guidelines.

After Institutional Ethics Committee approval and patient informed oral consent were obtained, adult patients scheduled for elective open chest cardiac surgery in the Clinique SaintLuc of Bouge (Namur, Belgium) were enrolled prospectively (Fig 1). The surgeries performed were coronary artery bypass graft, aortic or mitral valve replacement, combination of coronary artery bypass graft and valve replacement, and ascendant aneurysm repair (Table 1). Exclusion criteria were patients who were not in regular sinus rhythm and patients with 1 or more vasopressor when measurements were performed. Therefore, none of the patients received vasoactive drugs during the measurements. Surgery was preceded by a 6-hour fasting period. All patients were monitored (electrocardiogram, pulse oximetry, invasive blood pressure, neuromuscular monitoring, airway pressure, and capnograph) using the Datex-Ohmeda S/5 (Datex-Ohmeda, Helsinki, Finland). Patients underwent placement of a $20 \mathrm{G}$ radial arterial catheter (BD Angiocath; Becton, Dickinson and Company, Franklin Lakes, NJ). General anesthesia was induced using propofol $(1 \mathrm{mg} / \mathrm{kg})$, midazolam $(0.1 \mathrm{mg} / \mathrm{kg})$, and sufentanil $(0.1 \mathrm{mg} / \mathrm{kg})$. Neuromuscular relaxation was achieved using cisatracurium $(0.15 \mathrm{mg} / \mathrm{kg})$. Patients were intubated with a single lumen orotracheal tube and mechanically ventilated (volume control, tidal volume $\geq 8 \mathrm{~mL} / \mathrm{kg}$ lean body weight) with the Aisys $\mathrm{CS}^{2}$ (DatexOhmeda). Anesthesia was maintained using sevoflurane and sufentanil $(0.05 \mathrm{mg} / \mathrm{kg} / \mathrm{h})$.

An anesthesiologist (PG \& JC) recorded patients' characteristics (age, sex, weight, height, type of surgery, and American Society of Anesthesiologists physical status); the amount of volume perfused before sternotomy; and the tidal volume during measurements of DD and DPP.

As the authors described previously, for the DD and DPP calculations, the systemic arterial pressure input signal was recorded as a "pulmonary arterial pressure" signal on the monitor (Datex-Ohmeda S/5), allowing the anesthesiologist to freeze the arterial curve (the closed pulmonary arterial pressure screen that provides the arterial pressure and the upper airway pressure simultaneously). For the sake of precision, the upper limit of the scale of this "pulmonary" pressure was set at $30 \mathrm{mmHg}$ more than the actual systolic arterial pressure. ${ }^{9}$

Pulse pressure is the difference (in $\mathrm{mmHg}$ ) between the systolic and diastolic pressure. The DPP is calculated as the ratio of the differential between the maximal and the minimal pulse pressure values during 1 breathing cycle, divided by their mean (Fig 2). ${ }^{9}$ The DD is defined as the difference between the last systolic pressure before insufflation (lung inflation, inspiratory phase) and the lowest systolic value during the expiratory phase (at the end of a $5 \mathrm{~s}$ expiratory pause) (see Fig 2). ${ }^{14}$

During DD and DPP acquisitions, the tidal volume was set at a value $>8 \mathrm{~mL} / \mathrm{kg}$ (see Table 1) and the ventilation rate was reduced to 6 minutes to mimic the conditions of a respiratory pause, as recommended for the measurements of DD. ${ }^{8,9}$ No positive end-expiratory pressure was applied during measurements.

For all patients, a second anesthesiologist (PG \& JC) measured both DD and DPP just before sternotomy (DDb and $\mathrm{DPPb}$ ) and the same 2 measurements 10 minutes later after placement of sternal retractors and creation of a pericardial incision (but without a pleura incision, which occurs later than 10 minutes after sternotomy in the authors' institution) (DDs and DPPs). DD and DPP measurements were calculated offline by a third investigator (SD) using an automated Microsoft Excel (Microsoft, Redmond, WA) spreadsheet. All fluid infusions were stopped during the time interval between the 2 sets of measurements (before and after sternotomy). Patients were excluded in case of arrhythmias after sternotomy, if a vasopressor was needed, if variation in blood pressure (systolic and diastolic) was more than $10 \%$ before and after sternotomy (to exclude sympathetic response due to sternotomy), and if massive bleeding occurred during sternotomy. This approach mitigated the effect of volume changes on the measurements. Tidal volume was kept equal for the 2 measurements of DD and DPP. 


\section{ENROLLMENT}

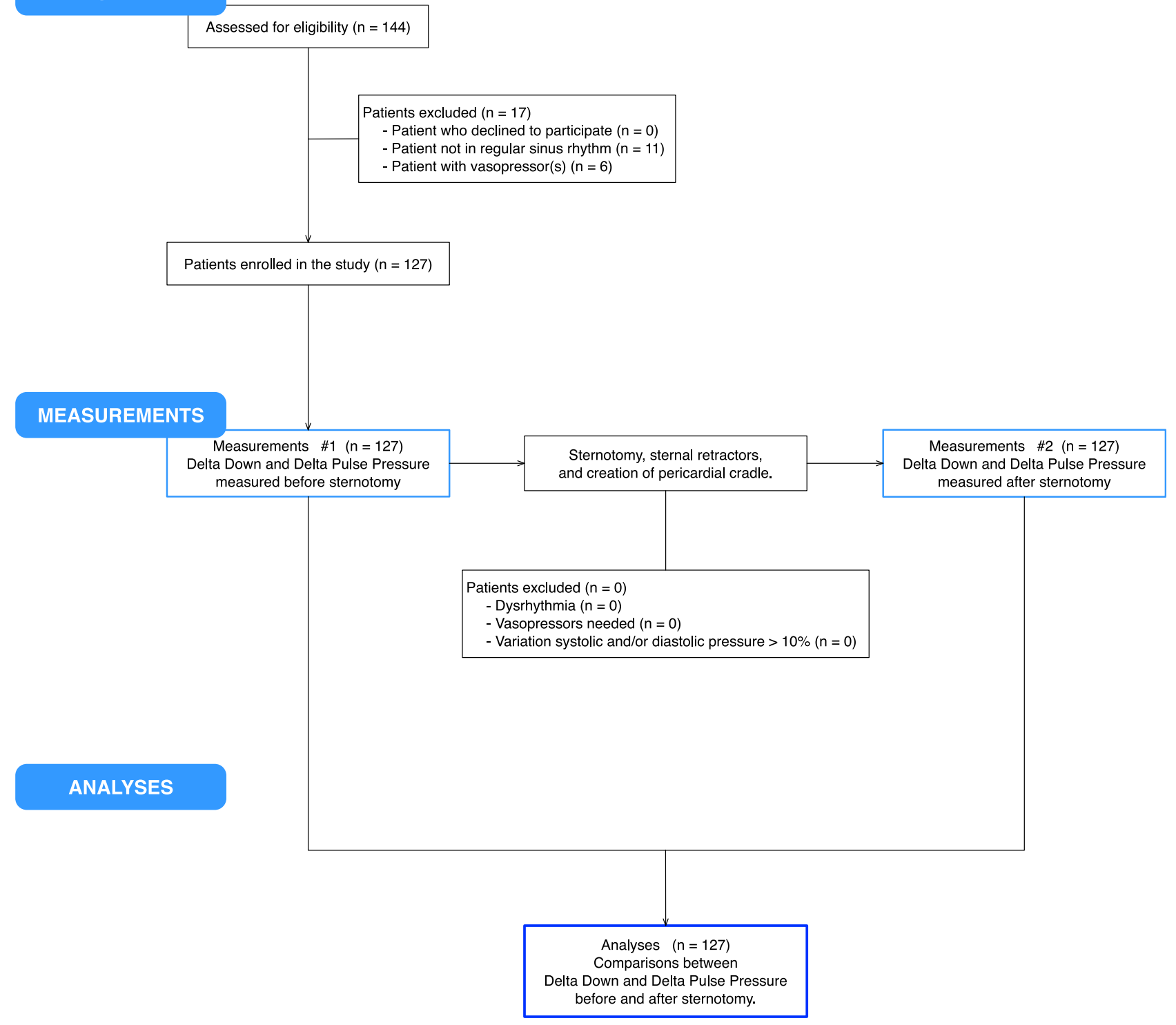

Fig 1. Flowchart.

\section{Statistical Analysis}

A power sample size calculation estimated that a population of 111 patients would provide a $95 \%$ power for detecting an effect size of 0.3 at an alpha level of 0.05 .

The normality of distribution was assessed using a ShapiroWilk test.

First, DD and DPP were compared before and after sternotomy using the Mann-Whitney $U$ test.

Second, the same comparisons were performed between $\mathrm{DDb}$ and DDs and between DPPb and DPPs using the BlandAltman analysis. If the confidence interval (CI) (mean \pm 1.96 standard deviation) of the mean difference between measures differed from 0 , a significant difference between the 2 methods existed. ${ }^{15}$ This analysis allows for the determination of possible bias in measurements induced by sternotomy.
Third, for patients who had a DD $>5 \mathrm{mmHg}$ before sternotomy, the authors calculated the number who still had a DD $>5 \mathrm{mmHg}$ after sternotomy. The same measures were achieved for patients who had a DPP $>13 \%$ before sternotomy. A comparison between the measures before and after sternotomy were performed using the McNemar test.

Fourth, DD and DPP were modified into a binary system (0-1) for which DD was $<5 \mathrm{mmHg}(0)$ or $>5 \mathrm{mmHg}$ (1) and DPP was $<13 \%$ (0) or $>13 \%$ (1). This binary system allowed for the comparison of DD and DPP before and after sternotomy using the kappa coefficient of Cohen (interobserver agreement). The binary DDb was compared with the binary DDs and the binary DPPb with the binary DPPs. The kappa value can be interpreted as follows: $<0.20$ corresponds to a poor agreement, 0.21 to 0.40 to a fair agreement, 0.41 to 0.60 to a moderate agreement, 0.61 to 0.80 to a good agreement, and 0.81 to 1.00 to a very good agreement. ${ }^{16}$ 
Table 1

Demographic and Perioperative Characteristics of Patients Enrolled in the Study Population

\begin{tabular}{ll}
\hline & $\mathrm{n}=127$ \\
\hline Age & $67.19(11.33)$ \\
Male/female $(\%)$ & $79.53 / 20.47$ \\
Height $(\mathrm{cm})$ & $171.9(8.55)$ \\
Weight $(\mathrm{kg})$ & $82.69(15.3)$ \\
BMI $(\mathrm{kg} / \mathrm{m})$ & $27.94(4.61)$ \\
ASA physical status $(\%)$ 1/2/3/4 & $0 / 0 / 96.85 / 3.15$ \\
Type of surgery $(\%)$ & \\
CABG & 71.65 \\
VR & 13.37 \\
CABG + VR & 9.45 \\
AAR & 5.53 \\
Tidal volume for the measurements (mL/kg) & $8.21(8.10-8.42)$ \\
Amount of infusion given before sternotomy (mL) & \\
Total & $500(250-600)$ \\
Crystalloid & $250(0-500)$ \\
Colloid & $200(0-500)$ \\
\hline
\end{tabular}

NOTE. Data are presented as mean (standard deviation), percentage, or median (interquartile range).

Abbreviations: AAR, ascendant aneurysm repair; ASA, American Society of Anesthesiologists; BMI, body mass index; CABG, coronary artery bypass graft surgery; VR, valve replacement.

Data were analyzed using XLSTAT for Mac (Addinsoft SARL, V 18.07.39180; Paris, France); NCSS 12 Statistical Software (2018) (NCSS, LLC, Kaysville, UT); and MedCalc Statistical Software, version 18.2.1 (MedCalc Software bvba, Ostend, Belgium). A 2-tailed $\mathrm{p}$ value $<0.05$ was considered to be statistically significant.

\section{Results}

One hundred and forty-four patients met the inclusion criteria. Seventeen patients were excluded before surgery (patients who were not in regular sinus rhythm $[n=11]$ and patients who necessitated the use of 1 or more vasopressors $[n=6]$; see
Fig 1). No patients declined to participate in the study. One hundred and twenty-seven patients were finally enrolled. No patients were excluded after inclusion into the study. Table 1 presents demographic and perioperative characteristics.

First, the Mann-Whitney test demonstrated that there was no significant difference between DD before and after sternotomy (median [interquartile range]: $\mathrm{DDb}=2[1-4], \mathrm{DDs}=2[1-4]$; $\mathrm{p}=0.528$ ). Conversely, the same type of analysis demonstrated that DPP was significantly influenced by sternotomy: $\mathrm{DPPb}=6.06$ (2.76-10.7), DPPs $=4.51$ (2.03-8.74); $\mathrm{p}=0.028$.

Second, the Bland-Altman analysis revealed that the mean difference between DDb and DDs was not significantly different from 0 (mean difference [95\% CI] 0.173 [ -0.23 to 0.576 ; Fig 3, A). Based on this analysis, sternotomy did not induce a bias in DD measurement. Conversely, sternotomy induced a bias in DPP values. Indeed, the mean difference between $\mathrm{DPPb}$ and DPPs was significantly different from $0(1.765$ [0.621-2.91]; Fig 3, B). Regarding the Bland-Altman analyses, DD exhibited significantly better accuracy than did DPP between presternotomy and poststernotomy values.

Third, 24 patients $(18.9 \%)$ had a DD $>5 \mathrm{mmHg}$ before sternotomy. Of these 24 patients, 19 (14.9\%) had still a DD >5 $\mathrm{mmHg}$ after sternotomy. The McNemar test indicated that a positive DD was not influenced by the sternotomy $(\mathrm{p}=0.22)$. Twenty patients $(15.7 \%)$ had a DPP $>13 \%$ before sternotomy. Of these 20 patients, 7 (5.5\%) still had a DPP $>13 \%$ after sternotomy. The McNemar test indicated that a positive DPP was influenced by the sternotomy $(p=0.02)$. A McNemar test also was performed between DD and DPP before sternotomy (DDb and $\mathrm{DPPb})$. This test revealed no significant difference between the 2 measurements $(p=0.34)$. The same type of analysis was performed between the DD and DPP values obtained after sternotomy. It exhibited a significant difference between DD and DPP $(\mathrm{p}=0.01)$. The $4 \mathrm{McNemar}$ analyses revealed that sternotomy influenced DPP but not DD.

Fourth, the kappa coefficient of Cohen applied to the DD and DPP and modified into a binary system are illustrated in Figure 4 . The kappa (95\% CI) value between DD before and
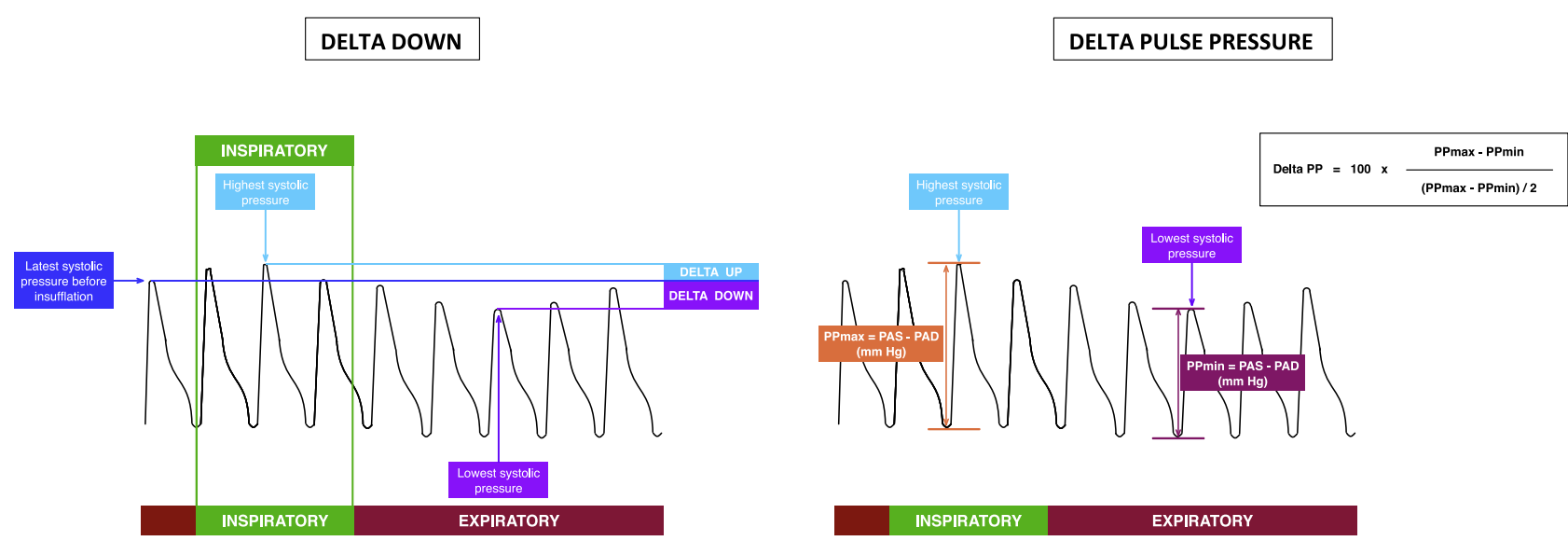

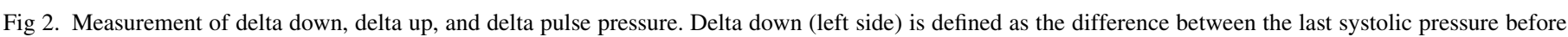

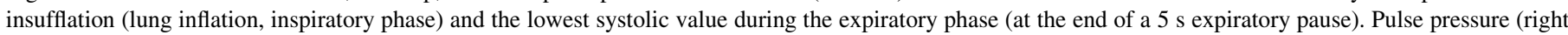

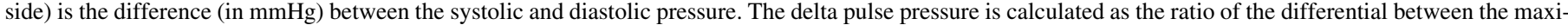
mal and minimal pulse pressures during 1 breathing cycle, divided by their mean. PAD, pressure arterial diastolic; PAS, pressure arterial systolic. 
A

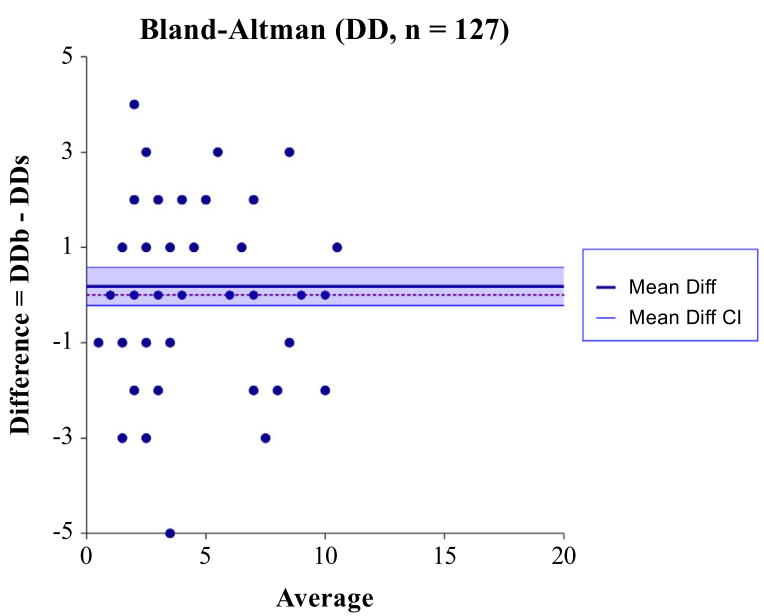

Units $\mathrm{x}$ - and y-axis: $\mathrm{mmHg}$

Mean difference $[95 \% \mathrm{CI}]: 0.173[-0.23$ to 0.576$] \mathrm{mmHg}$
B

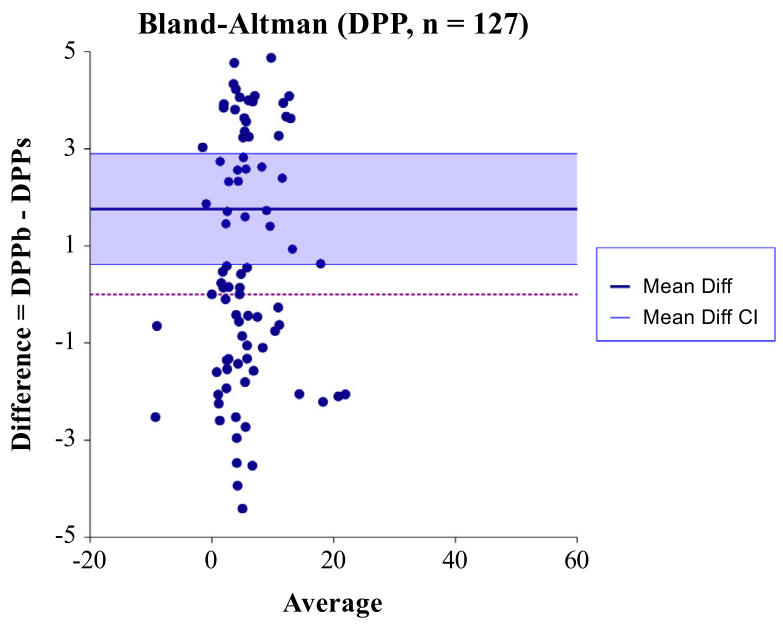

Units $\mathrm{x}$ - and $\mathrm{y}$-axis: \%

Mean difference $[95 \% \mathrm{CI}]: 1.765[0.621$ to 2.91$] \%$

Fig 3. Bland-Altman analyses. (A) Analysis of delta down before and after sternotomy. (B) Analysis of delta pulse pressure before and after sternotomy. Dotted red lines illustrate zero value in the y-axis; bold blue lines illustrate the mean difference; blue zones illustrate the $95 \%$ confidence interval of the mean difference. Results are significant at a $\mathrm{p}$ value $<0.05$ if the $95 \%$ confidence interval of the mean difference did not include zero value of the y axis. CI, confidence interval; DD, delta down; DDb, delta down before sternotomy; DDs, delta down after sternotomy; DPP, delta pulse pressure; DPPb, delta pulse pressure before sternotomy; DPPs, delta pulse pressure after sternotomy.

after sternotomy was $0.835(0.708-0.963)$ (very good agreement). The kappa value between DPP before and after sternotomy was 0.404 (0.173-0.635) (fair agreement). A $z$ test was applied to compare these 2 kappa coefficients and showed a significant difference between them ( $z$ value 6.12; $\mathrm{p}<0.001$ ).

Table 2 provides the detailed hemodynamic values at the time of DD and DPP measurements. The results indicate that heart rate and central venous pressure remained stable before and after sternotomy. Conversely, systolic and diastolic blood pressure were significantly greater after sternotomy. A

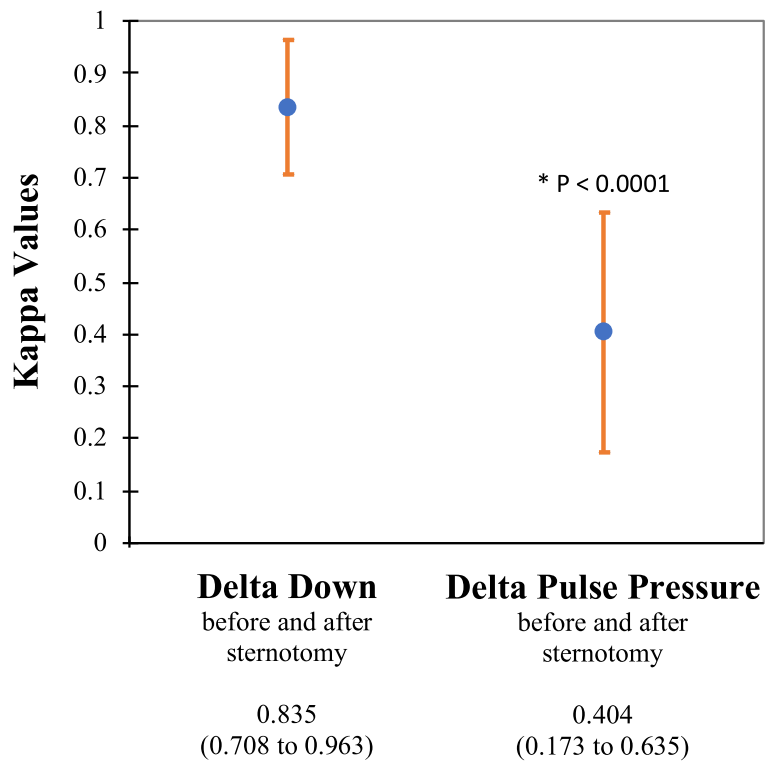

Fig 4. Kappa coefficients of Cohen and their $95 \%$ confidence interval. Kappa values on the left illustrate the agreement between delta down before and after sternotomy. Kappa values on the right illustrate the agreement between delta pulse pressure before and after sternotomy. sympathetic activation could not be excluded to explain this elevation, even if it is doubtful given the absence of a change in heart rate.

\section{Discussion}

The present study demonstrates that, in the study population, sternotomy significantly influences the DPP but not the DD. Previously, the authors validated the correlation between DD and DPP in closed chest surgery. ${ }^{9}$

Goal-directed hemodynamic therapy is challenging for anesthesiologists, but data in open chest cardiac surgery are poor. Piccioni et al. demonstrated that published studies reporting on this topic were not homogenous in the study protocol. This inhomogeneity can lead to contradictory results. ${ }^{10}$ Some authors have investigated the DPP under open thoracic conditions. $^{10-13}$ Some of these studies had a small sample size, and the studies show a wide variation in their results. Most of them conclude that DPP should not be used in patients after a sternotomy. ${ }^{10}$ Jeong et al. demonstrated that stroke volume variation and DPP seem to be inaccurate in predicting fluid responsiveness in open chest surgery. ${ }^{12}$ However, the agreement between DPP or DD values before and after sternotomy without any change in volemia has never been assessed.

Recently, it was demonstrated that a restricted fluid therapy (in abdominal surgery) was associated with an increased risk of acute kidney injury. ${ }^{4}$ This demonstrates, if necessary, the importance of goal-directed fluid optimization.

One strength of the present study resides in its methodology, which compared 2 measurements of both DD and DPP separate from a maximum of 10 minutes and avoiding any situation that can modify volemia. For example, vasoactive drugs may affect dynamic indicators. ${ }^{17}$ For this reason, the only variable 
Table 2

Detailed Hemodynamic Values at the Time of DD and DPP Measurements

\begin{tabular}{|c|c|c|c|c|}
\hline & & Before Sternotomy & After Sternotomy & $\mathrm{p}$ Value \\
\hline \multirow[t]{5}{*}{ General } & $\mathrm{SBP}(\mathrm{mmHg})$ & $105.85 \pm 18.59$ & $124.02 \pm 19.16$ & $<0.0001^{*}$ \\
\hline & $\mathrm{DBP}(\mathrm{mmHg})$ & $57.94 \pm 13.03$ & $66.12 \pm 12.13$ & $<0.0001^{*}$ \\
\hline & $\mathrm{MAP}(\mathrm{mmHg})$ & $73.91 \pm 14.18$ & $78.54 \pm 12.61$ & $<0.0001^{*}$ \\
\hline & $\mathrm{CVP}(\mathrm{mmHg})$ & $7.96 \pm 3.76$ & $7.24 \pm 4.08$ & 0.147 \\
\hline & HR (beats/min) & $64.65 \pm 13.66$ & $64.24 \pm 9.41$ & 0.103 \\
\hline \multirow[t]{2}{*}{ Delta down } & Latest SBP value before insufflation $(\mathrm{mmHg})$ & $103.78 \pm 18.9$ & $121.65 \pm 18.96$ & $<0.0001^{*}$ \\
\hline & Lowest SBP (mmHg) & $100.46 \pm 18.31$ & $118.51 \pm 19.09$ & $<0.0001^{*}$ \\
\hline \multirow[t]{4}{*}{ Delta pulse pressure } & Highest SBP (mmHg) & $105.85 \pm 18.59$ & $124.02 \pm 19.16$ & $<0.0001^{*}$ \\
\hline & Lowest SBP (mmHg) & $100.46 \pm 18.31$ & $118.51 \pm 19.09$ & $<0.0001^{*}$ \\
\hline & Highest DBP $(\mathrm{mmHg})$ & $57.94 \pm 13.03$ & $66.12 \pm 12.13$ & $<0.0001^{*}$ \\
\hline & Lowest DBP (mmHg) & $55.66 \pm 12.57$ & $63.51 \pm 12.09$ & $<0.0001^{*}$ \\
\hline
\end{tabular}

NOTE: Data are expressed as mean \pm standard deviation.

Abbreviations: CVP, central venous pressure; DBP, diastolic blood pressure; HR, heart rate; MAP, mean arterial pressure; SBP, systolic blood pressure

$*$ p value $<0.05$ was considered to be significant.

between the 2 DD values and the 2 DPP values consists of the sternotomy. To the best of the authors' knowledge, such methodology has never been applied.

The present study corroborates previous results indicating that DPP is significantly influenced by sternotomy. ${ }^{10,12}$ Surprisingly, it seems that sternotomy does not influence DD. The authors presume that this difference with DPP may reside in the fact that DD does not integrate the delta up component (see Fig 2). Delta up is defined as the difference between the latest systolic pressure before insufflation and the higher systolic pressure (occurring during the inspiratory cycle). Delta up corresponds to an augmentation of the left ventricle preload during positive pressure ventilation. This increase relates to an in-rush of blood from the pulmonary capillary to the left side of the heart. This phenomenon is dependent on the positive pressure inside the lung (due to insufflation). The sternotomy could minimize this effect and thus would make delta up less reliable. DPP calculation considers the blood sequestered in the pulmonary bed ("delta up"), whereas DD is independent of this parameter. The authors hypothesize that this difference could be an explanation for the disparity of agreement between DD and DPP before and after sternotomy. However, it is only a hypothesis and must be verified in future studies.

Methods of calculation also are different between DD and DPP. DD takes into account only the variation of systolic pressure, whereas DPP integrates the differential between the systolic and diastolic pressures. A hypothesis could be that the diastolic pressure is more sensitive to sternotomy than the systolic pressure. However, the authors do not have any physiological explanation for that. Additional studies on a larger scale should verify these 2 hypotheses.

The present study presents 3 limitations. First, no cardiac function measures (ejection fraction, cardiac output, stroke volume variation) were recorded. Second, the study was purely observational and conducted at 1 institution. The aim of the study simply was probing whether DD or DPP changes after sternotomy; therefore the authors did not examine any measure of volume responsiveness. This point must be investigated in future studies before widely promoting the use of DD in open chest surgery. Future studies will need to focus on the concordance between DD, DPP, and cardiac function measurements and the effects of fluid challenge on these different parameters. Finally, a sympathetic activation after sternotomy could not be dismissed, even if it is unlikely.

In conclusion, with the methodology described herein, the authors confirmed that sternotomy influences DPP. In the present study's population, DD appears to be more accurate and independent of the influence of sternotomy. However, because these are the first data indicating this agreement, the results must be confirmed on a larger scale and mainly in a study assessing fluid responsiveness to fluid challenge. This study was a preliminary study.

\section{References}

1 Grocott MP, Mythen MG, Gan TJ. Perioperative fluid management and clinical outcomes in adults. Anesth Analg 2005;100:1093-106.

2 Chong MA, Wang Y, Berbenetz NM, et al. Does goal-directed haemodynamic and fluid therapy improve peri-operative outcomes? A systematic review and meta-analysis. Eur J Anaesthesiol 2018;35:469-83.

3 Malbouisson LMS, Silva JM Jr., Carmona MJC, et al. A pragmatic multicenter trial of goal-directed fluid management based on pulse pressure variation monitoring during high-risk surgery. BMC Anesthesiol 2017;17:70.

4 Myles PS, Bellomo R, Corcoran T, et al. Restrictive versus liberal fluid therapy for major abdominal surgery. N Engl J Med 2018;378:2263-74.

5 Michard F. Changes in arterial pressure during mechanical ventilation. Anesthesiology 2005;103:419-28.

6 Marik PE, Baram M, Vahid B. Does central venous pressure predict fluid responsiveness? A systematic review of the literature and the tale of seven mares. Chest 2008;134:172-8.

7 Michard F, Chemla D, Richard C, et al. Clinical use of respiratory changes in arterial pulse pressure to monitor the hemodynamic effects of PEEP. Am J Respir Crit Care Med 1999;159:935-9.

8 Michard F, Boussat S, Chemla D, et al. Relation between respiratory changes in arterial pulse pressure and fluid responsiveness in septic patients with acute circulatory failure. Am J Respir Crit Care Med 2000;162:134-8.

9 Deflandre E, Bonhomme V, Hans P. Delta down compared with delta pulse pressure as an indicator of volaemia during intracranial surgery. Br J Anaesth 2008;100:245-50. 
10 Piccioni F, Bernasconi F, Tramontano GTA, et al. A systematic review of pulse pressure variation and stroke volume variation to predict fluid responsiveness during cardiac and thoracic surgery. J Clin Monit Comput 2017;31:677-84.

11 Lorne E, Mahjoub Y, Zogheib E, et al. Influence of open chest conditions on pulse pressure variations. Ann Fr Anesth Reanim 2011;30:117-21.

12 Jeong DM, Ahn HJ, Park HW, et al. Stroke volume variation and pulse pressure variation are not useful for predicting fluid responsiveness in thoracic surgery. Anesth Analg 2017;125:1158-65.

13 Reuter DA, Goepfert MS, Goresch T, et al. Assessing fluid responsiveness during open chest conditions. Br J Anaesth 2005;94:318-23.
14 Perel A, Pizov R, Cotev S. Systolic blood pressure variation is a sensitive indicator of hypovolemia in ventilated dogs subjected to graded hemorrhage. Anesthesiology 1987;67:498-502.

15 Bland JM, Altman DG. Statistical methods for assessing agreement between two methods of clinical measurement. Lancet 1986;1:307-10.

16 Landis JR, Koch GG. An application of hierarchical kappa-type statistics in the assessment of majority agreement among multiple observers. Biometrics 1977;33:363-74.

17 Gelman S, Bigatello L. The physiologic basis for goal-directed hemodynamic and fluid therapy: The pivotal role of the venous circulation. Can J Anaesth 2018;65:294-308. 